•综述・

\title{
海洋生境制图研究进展: 概念、方法与应用
}

\author{
胡文佳 ${ }^{1,3}$, 周秋麟 ${ }^{1}$, 陈涁 ${ }^{1,3^{*}}$, 杨圣云 ${ }^{2}$, 肖佳媚 ${ }^{2}$, 杜建国 ${ }^{(1,3}$, 黄浩 ${ }^{1,3}$, 俞炜炜 ${ }^{1,3}$, \\ 马志远 1,3
}

1. 自然资源部第三海洋研究所, 福建厦门 361005; 2. 厦门大学海洋与地球学院, 福建厦门 361005; 3. 福建省海洋生态保护与修复重点实 验室, 福建厦门 361005

摘要: 基于生态系统的海洋管理采用生态边界而非行政边界来指导管理实践, 海洋生境制图旨在探寻海洋环境与生物群落/ 物种的分布规律, 从而为生物多样性保护和海洋空间规划等管理行动提供可以依循的生态边界。海洋生境制图是科研监测数 据通往管理决策应用的有效途径, 近年来已成为海洋生态保护与管理领域的国际研究热点, 但我国对于海洋生境制图的研究 才刚刚起步。本文对海洋生境制图的国内外研究进展进行了综述, 介绍了海洋生境制图的定义和内涵, 明确了海洋生境制图 的步骤及其在生态系统管理中的应用; 系统阐述了海洋生境制图的主要研究方法, 从生境分类方案、生境分区和生态特征描 述等方面对海洋生境制图的研究过程及采用的分析方法进行了总结; 全面梳理了国内外的代表性海洋生境制图研究案例, 对 各国已开发的海洋生境分类分区体系进行了对比和评述，总结了共性特征; 最后提出研究建议, 对我国开展海洋生境制图研 究的前景进行展望, 以期为我国的海洋生态空间管理工作提供科学依据和参考。

关键词: 生境制图; 海洋空间规划; 生境分类; 生态分区

胡文佳, 周秋麟, 陈涁, 杨圣云, 肖佳媚, 杜建国, 黄浩, 俞炜炜, 马志远 (2021) 海洋生境制图研究进展: 概念、方法与应用. 生物多样性, 29, 531-544. doi: 10.17520/biods.2020176.

Hu WJ, Zhou QL, Chen B, Yang SY, Xiao JM, Du JG, Huang H, Yu WW, Ma ZY (2021) Progress in marine habitat mapping: Concept, methods, and applications. Biodiversity Science, 29, 531-544. doi: 10.17520/biods.2020176.

\section{Progress in marine habitat mapping: Concept, methods, and applications}

Wenjia $\mathrm{Hu}^{1,3}$, Qiulin $\mathrm{Zhou}^{1}$, Bin Chen ${ }^{1,3^{*}}$, Shengyun Yang${ }^{2}$, Jiamei Xiao ${ }^{2}$, Jianguo Du ${ }^{1,3}$, Hao Huang ${ }^{1,3}$, Weiwei Yu ${ }^{1,3}$, Zhiyuan $\mathrm{Ma}^{1,3}$

1 Third Institute of Oceanography, Ministry of Natural Resources, Xiamen, Fujian 361005

2 College of Oceanic and Earth, Xiamen University, Xiamen, Fujian 361005

3 Fujian Provincial Key Laboratory of Marine Ecological Conservation and Restoration, Xiamen, Fujian 361005

\section{ABSTRACT}

Context: The ecological boundaries rather than the administrative boundaries are adopted in the Marine EcosystemBased Management (MEBM) guide to management practices. Marine habitat mapping aims to explore the distribution patterns of the marine environment, biomes, and species. This provides ecological boundaries for management actions like biodiversity conservation and marine spatial planning.

Aims: Marine habitat mapping is an effective approach in using monitoring data for decision-making, which has become popular for research focused on marine ecosystem conservation and management. However, marine habitat mapping has only just begun in China. This article aims at reviewing domestic and foreign research progress of marine habitat mapping.

Progresses: First, we explain the definition and connotation of marine habitat mapping. Then, we clarify the steps of marine habitat mapping and its applications for ecosystem-based management. The methods for marine habitat mapping were systematically summarized based on the aspects of habitat classification scheme, ecoregion delineation, and characteristics descriptions. Furthermore, we summarize the representative marine habitat mapping cases. By comparing the marine habitat mapping systems developed by different countries/regions, we proposed common

收稿日期: 2020-04-29; 接受日期: 2020-08-18

基金项目：科技部国家重点研发计划(2018YFC0507205; 2017YFC0506105)和国家自然科学基金(41906127; 42076163)

* 通讯作者 Author for correspondence. E-mail: chenbin@tio.org.cn 
characteristics across different systems. Finally, we present suggestions in interpreting the prospects of marine habitat mapping applications in China, providing scientific basis for China’s marine ecological spatial management.

Key words: habitat mapping; marine spatial planning; habitat classification; ecological regions

生态系统是植物、动物、微生物群落及其生存 环境作为一个生态单位交互作用形成的动态复合 体(Cropper, 1993)。在一定海域内, 海洋生物群落与 周围环境相互作用构成了具有相对稳定功能并能 自我调控的生态单元, 其中在特定时空尺度上可通 过非生物特征和其中的生物聚落来区分的空间称 为生境(ICES, 2005)。海洋生境制图(marine habitat mapping)研究能够揭示海洋环境和海洋生物群落的 地理分异规律, 划定不同尺度的海洋生物地理区, 同时识别各类生境之间空间、时间的分界, 以及它 们相互之间的联系。

为推动海洋重要物种、生物群落、典型生态系 统和渔业资源的保护与恢复, 以及海洋生态系统健 康与风险的科学评估、监控和管理, 需要解决海洋 生态系统类型重叠、生物群落空间分布不明、管理 边界不确定等问题(Gray, 1997; Bland et al, 2017), 海洋生境制图在这方面为科研监测数据通往管理 决策应用提供了有效的途径和工具。但海洋生境制 图研究的发展滞后于陆地同类研究, 仅有约 5\%-10\%的全球海底生境以与陆地研究近似的分辨 率绘制(Wright \& Heyman, 2008), 此外, 海洋生态 系统的描述程度也较低, 据估计陆地未知生境的比 例约为 $17 \%$, 而在海洋领域则约为 $40 \%$ (European Commission, 2007)。因此, 国际海洋考察理事会 (International Council for the Exploration of the Sea, ICES)发布的《海洋战略框架指令》(Marine Strategy Framework Directive)基于底栖生境保护、海洋空间 规划、人类活动对海洋影响的空间估算等管理行动 的迫切需求, 已将海洋生境地图列为海洋管理和研 究中必不可少的项目(Strong et al, 2019)。目前, 国 际上已形成从全球尺度到国家区域尺度的多种海 洋生境分类方案和分区地图(Connor et al, 2004; Spalding et al, 2007; UNESCO, 2009; FGDC, 2012; Populus et al, 2017), 在各国的生物多样性保护和海 洋国土空间管理中发挥了关键作用, 但我国鲜有这 方面的研究探索。

为掌握我国海洋生态系统现状、支撑空间化的 海洋生态保护与管理行动, 需要开展海洋生境制图
研究, 从而科学确定海洋生态单元的独立空间, 明 确各类生态单元的主导生态特征和生物资源。长期 以来, 我国在各类海洋专项调查中获取了大量的观 测数据, 但缺乏有效工具将不同的海洋生物和环境 观测数据进行科学的整合评估, 实现从科学调查到 管理实践的衔接。通过开展海洋生境制图研究, 能 够对调查获取的海量数据进行系统整理及信息可 视化, 从而促进对海洋珍稀物种、关键生物群落和 重要生态系统的监测、评估和保护的科学认知, 推 进以生态系统为基础的自然资源管理。因此, 本文 对海洋生境制图的国内外研究进展进行综述, 对海 洋生境制图的定义和步骤进行介绍, 系统阐述海洋 生境制图的主要研究方法, 总结各国已开发的海洋 生境分类体系和案例, 最后提出未来的研究方向, 以期为我国开展海洋生境分类制图的相关工作提 供科学依据与参考。

\section{海洋生境制图的定义与步骤}

\section{1 海洋生境制图的定义和内涵}

整合海洋生物和环境等生境信息, 辅以遥感技 术和空间分析手段, 将所收集的数据解释并转换为 有生态意义的地图的过程被称为海洋生境制图, 其 已成为国际上通用的生态系统管理工具之一(Ball et al, 2006)。国际上对海洋生境制图尚没有公认的 定义, 这可能是由于相关研究的目的各不相同, 不 同环境和生物要素分布特征的区别决定了生境制 图的具体内容及其展现形式的差异(Diaz et al, 2004)。在欧盟的海洋生境制图计划中, 海洋生境制 图被定义为“绘制生境的分布和范围, 以形成完整 覆盖海底并显示相邻生境间的清晰分隔边界的地 图” (Buhl-Mortensen et al, 2015a), 目前该定义的采 用程度相对较高。

从字面定义看, 海洋生境制图的目标在于区分 海洋生境的边界, 但在看似简单的边界中内含海洋 生物和环境分布规律的复杂信息, 将生态系统组分 与空间地理格局进行了紧密的联系。在生态系统中, 物种沿环境梯度分布, 物种之间的相互作用(捕食、 共栖、共生和竞争等)导致一定环境条件下某些物种 
类别同时出现, 从而形成生物群落, 因此生物群落 的空间分布格局往往也与环境梯度密切相关(Gray, 1997)。海洋生境制图通过将环境因子和生物信息 (例如, 水深、地形、底质、水动力、生物群落组成 等)划入不同的生境类别, 假定每个类别都与独特 的非生物条件和可识别的生物群落相关联, 由此产 生具备环境或生态意义的空间单元(Strong et al, 2019)。海洋生境制图基于生态系统的理论, 从海洋 生态系统的整体性和连通性出发, 关注生物潜在分 布、结构特征和生态问题均相似的生态空间单元。

\section{2 海洋生境制图的步骤及其应用}

生态系统管理的特点是采用物种、群落或生态 系统分布的生态边界来指导海洋管理, 而不是行政 或政治边界(McLeod et al, 2005; Crowder \& Norse, 2008; Katsanevakis et al, 2011)。在海洋中, 由于水体 的覆盖使得管理者无法直接观察和确定生态边界, 故需通过海洋生境制图来标记并向管理者直观展 示这些生境的空间分布(Mumby \& Harborne, 1999)。

海洋生境制图通过在特定的分类系统内进行 分区绘制来识别空间单元, 并在特定尺度下以适当 的比例表征生态空间单元，一般包括三个步骤: (1) 数据收集。除文献资料数据外, 通常还包括两类主 要数据集: 全覆盖的多变量空间数据集; 底质或生 物群落的站位记录数据集(Buhl-Mortensen et al, 2015b)。(2)针对研究或应用目标提出生境分类方案 (habitat classification scheme)。生境分类方案提供了 一个结构化的框架, 旨在借助归类过程将具有不同 空间比例尺、单位和格式的环境与生物信息统一至 具有生态意义的决策节点, 集成为最终的成果 (Costello, 2009)。(3)生境分区及特征描述。在生境 分类的框架下通过各种技术方法将收集的文献资 料数据、离散样本数据和连续变量数据分割为生态 相关的空间单位, 从而生成海洋生境地图, 并对划 分出的空间单元内的环境生物特征及其生态意义 予以定义和描述(Strong et al, 2019)。海洋生境地图 的形式可以是生物地理分区图、底栖物种或群落分 布图、典型生态系统分布图等。海洋生境制图的根 本目的是寻找物种、群落或生态系统分布的生态边 界, 在这三个步骤中, 生境分类方案和生境分区及 特征描述是海洋生境制图的核心内容, 前者是后者 的依据, 后者是前者的产品(Strong et al, 2019), 在 部分研究中, 这两者有时会同时进行。
在基于生态系统的海洋管理过程中, 海洋生境 制图及其相关工作往往位于最底层的阶段, 为其上 的各种决策和行动提供最基础的支持(Keefer et al, 2008; Cogan et al, 2009) (图1)。例如, 在全球尺度上 海洋生境制图的代表性示例包括全球大洋和深海 海底生物地理分类(UNESCO, 2009)和沿海大陆架 “世界海洋生态区” (Spalding et al, 2007)等, 在这些 分区框架下, 可以使用统一的生态边界和分区来支 持特定的管理活动(ICES, 2008; Schiele et al, 2015)。 经研究和实践, 海洋生境制图可为解决以下应用需 求提供关键支持: (1)海洋空间规划, 包括管理实践 的有效性评估及其在保护生物多样性中的作用; (2) 生物多样性和海洋生态系统健康评估; (3)敏感生态 系统(深海珊瑚、海山、北极等) 以及珍稀或资料贵 乏物种的保护; (4)了解生态系统产品和服务对社会 经济的作用并预测人类活动的影响; (5)沿海栖息地 对资源物种种群动态的作用; (6)风能、潮汐能和波 能等可再生能源的开发对海洋生境和生物区系的 影响; (7)沿海地区污染物、富营养化和栖息地变化 对人口和社区的影响; (8)捕捞对海洋生态系统的影 响; (9)气候变化过程和影响预测; (10)外来种和入侵 种对生态系统的影响以及与气候变化过程的相互 作用(McGowan et al, 2013; Malcolm et al, 2016; Thiault et al, 2020; Ware \& Downie, 2020)。

\section{2 海洋生境制图的方法}

\section{1 海洋生境分类方案}

\subsection{1 海洋生境分类方案的内容}

海洋生境制图的过程和最终结果取决于具体 的生境分类方案, Robinson和Levings (1995)将生境 分类方案定义为识别、划定和描述具有不同生物组 合(群落或物种)的生境的指南。国际上针对海洋生 境分类方案已进行了不同程度的研究和探索, 目前 已有的大部分生境分类方案具有层次结构, 其中普 遍混合了地貌和生物的信息。

生境分类方案的具体内容与制图的空间范围 和精度有关, 选择恰当的目标和空间尺度十分重要, 这决定了生境分类方案需采用哪些生境信息, 类型 细分到什么层次。在较高级别的分类中, 尺度和类 型都较粗略, 而在较低的级别上, 分类则更趋向生 物学属性等更精细的分类(TFMPA ANZECC, 2000)。总体来看, 物理、化学和生物信息的相对重 


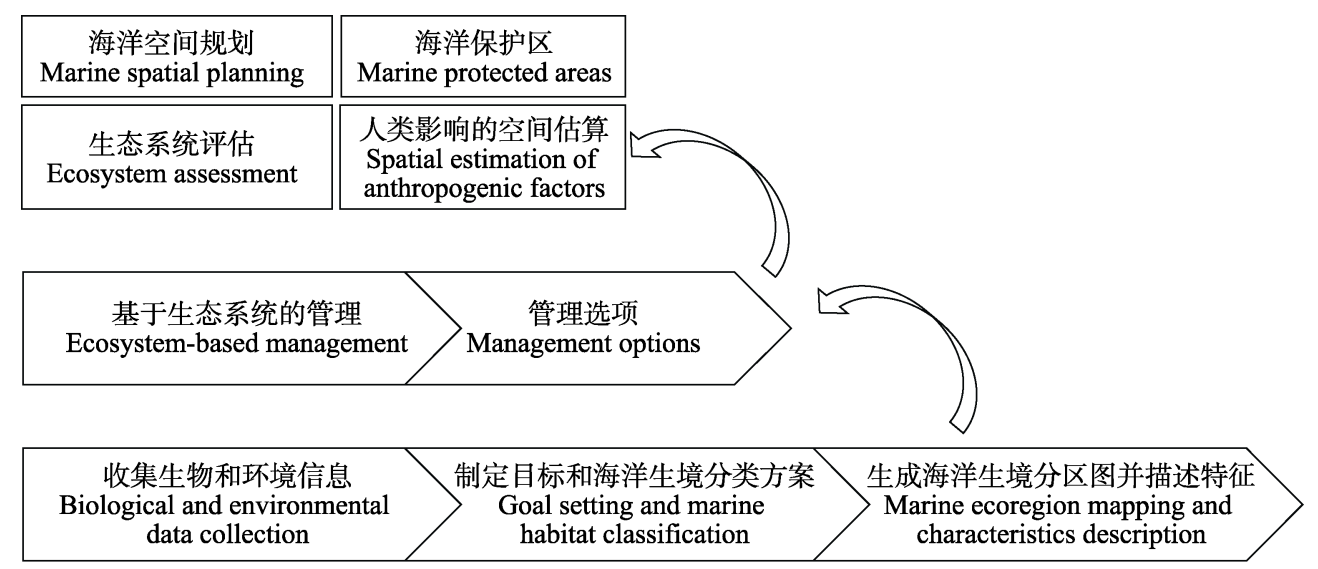

图1 海洋生境制图及应用步骤

Fig. 1 Stages of marine habitat mapping and application

要性会直接体现到生境单元分类的最终结果上, 其 中物理信息通常与分类层次结构的上层相关联, 往 往基于水动力模型和数字高程模型的大尺度低分 辨率数据进行分类。层次结构的较低级别可能描述 了生物群落和单个物种分布, 这需要生物数据并且 通常应用于更小的尺度。由于生物数据的不足, 某 些生境分类方案纯粹基于海底的物理和环境特征, 在假设非生物特征与生物群落分布之间具备相关 性的前提下，它们被用作生境的替代(Brown et al, 2011; Huang et al, 2011)。但亦有研究指出, 在仅采 用非生物因子进行生境分类的情况下, 只能解释 $30 \%$ 以下的生物群落相似性, 生境分类的误差在 20\%-62\%之间, 因此需要通过适当规模的生物学调 查对这类生境制图结果进行校准 (Stevens \& Connolly, 2004; Ramos et al, 2017)。

\subsection{2 海洋生境分类的因子选择}

过去海洋生境制图的发展滞后于陆地同类研 究, 这与海洋调查采样的难度有关。近几十年来, 随着科技的发展海洋环境和生物的采样技术取得 了长足的进步, 人类对海洋的监测已往更大的尺度 和更高的空间分辨率发展, 制定海洋生境分类方案 时所能获取的驱动因子信息越来越多(Boswarva et al, 2018)。目前海洋生境制图的采样方式可以分为 四大类: (1)声学、声呐技术; (2)光学遥感; (3)水下摄 影录像; (4)沉积物和生物采样(Harris \& Baker, 2020)。其中声呐技术主要用于水下地形探测, 可获 取水深、地形等因子的数据; 光学遥感技术通过收 集浅表海洋环境的光谱和影像信息, 可获取海表叶 绿素、海温等因子的时空分布; 水下摄影录像技术
用于收集水下静态或动态图像, 可直接对海底底质 和动植物分布进行观测和记录; 沉积物和生物采样 获得的样本可用于判断沉积物特性(例如粒径大小、 矿物类型等)和生物物种分类, 这些因子的确切信 息仅能通过实体样本获取。

虽然技术的进步驱动着海洋生境制图的进化, 但生境分类方案设计、空间分辨率和绘制尺度仍应 基于科学的需求(Cogan et al, 2009), 而非盲目追求 更高的精度或者更复杂的体系。目前人们尚无法对 所有海洋生物的存在及其空间分布具备完全精确 的了解, 难以绘制出海洋的真实生境地图。但是, 使用已有的数据信息和已知的生物环境关系, 推测 并大致区分生物和生态系统可能的空间分布是可 行的(Harris \& Baker, 2020)。所以, 从生态系统管理 的目标出发, 选择适当的驱动因子和分辨率, 用最 合理的分类对生境进行清晰的表征，才能够实现海 洋生境制图的高效应用。

因此, 在实际应用中, 虽然目前已提出了很多 复杂的生境分类方案，但各研究机构或政府部门往 往根据各自的区域特色和不同研究尺度选择主导 生物环境因子来进行分类 (Carey et al，2015; Livingstone et al, 2018), 以确保生境制图的可操作 性和实用性。在已有的各种生境分类中, 最主要的 依据包括地理隔离、水深和地形, 或遥感和海洋学 数据(Longhurst, 2007; Oliver \& Irwin, 2008; Silvia et al, 2013; Bailey, 2014), 有的研究则同时采用了海底 和水体变量(Harris \& Whiteway, 2009), 而在中小尺 度上, 底栖生物、大型藻类等生物调查数据可用于 对生境类型的细化、验证和描述(Fontaine et al, 2015; 
Ramos et al, 2017)。鉴于海洋环境和生物数据的获 取难度, 为保证制图和分区结果具备足够的科学依 据, 主导因子的选择可结合生物多样性分布和物种 生态位研究的结论来确定(Vassallo et al, 2018), 例 如有研究表明全球海洋生物的综合分布主要取决 于3个关键驱动因子: 水深、海温和光照强度(Gagne et al, 2020)。当然, 在有些尺度上溶解氧、底质等可 能也是重要因素(Mittermayr et al, 2020)。在数据有 限的情况下, 这些因子可以作为制定生境分类方案 时的优先选择。

\section{2 海洋生境分区和特征描述方法}

在确定生境制图所采纳的分类方案和驱动因 子后, 需对各类因子进行数据分析与评估, 从而根 据分类方案将海洋空间划分为管理所需的分区。目 前已有多项研究将全球海岸带、大陆架、深海和大 洋生境进行分区(表1), 方法主要有三类: (1)以文献 资料或调查结果获取的单一环境因子为主导要素 进行直接分区; (2)专家咨询法(德尔菲法); (3)多元 聚类分析法。其中后两类主要用于决定综合生物环 境因子下的分区, 在信息模糊或者难以进行直接采 样的情形下(例如深海)专家咨询法具有较大的优势, 但比起专家咨询法可能具备的主观认知限制, 目前 公认最全面和最客观的分区方法是多元聚类分析 法。数据聚类技术能够将相似的点、图斑或对象集 进行分组, 旨在将大型数据集划分为包含具有共同 特征元素的较小聚类, 因此十分适用于生物/生态 分区研究(Grant et al, 2006; Brown et al, 2011)。在多 元空间聚类分析法中, 近年来得到最广泛应用的方 法为K-均值聚类法(K-means clustering) (Livingstone et al, 2018)。K-均值聚类将数据(n)划分为多个预设 聚类 $(\mathrm{k})$, 同一个聚类内的数据点(在多元空间中)彼 此之间的相似性比其他聚类中的点更高, 从而实现 对海洋生境制图中多个生物环境因子的聚类分区。 伴随着海洋数据信息获取技术的发展, 这可能会成 为未来的主流分区方法。

在分区完成后, 需对分区或分区内的生境单元 特征进行描述。分区的大小和空间分辨率决定了能 够在生境地图上展示出哪些物理或生态特征, 以及 多少生态异质性。分区或生境单元内往往镶嵌或混 合了各种生物环境因子类型, 在描述过程中通常根 据优势类别来标记空间单位, 并附加相关次要生境 的信息作为修饰因子。特征描述方式主要有两种: (1)
采用统计图表对分区或生境单元内各类因子的范 围取值进行表征(Ramos et al, 2017; de la Hoz et al, 2018); (2)建立生境名称代码表, 将特征描述信息与 代码进行一一对应, 从而使用唯一的标识符表征特 定的生境信息, 例如群落构成、物种多样性、相对 丰度等(Sotheran et al, 2014; Carey et al, 2015; Bekkby et al, 2017)。前者适用于对大范围分区的环 境变量或特征信息进行直观的标示, 后者适用于对 复杂的生境分类因子进行标准化描述。

\section{国内外海洋生境制图研究案例}

\section{1 国外研究案例}

目前国际上较为成熟的海洋生境制图方案包 括两个全球性的大洋分区, 以及美国、加拿大、英 国及爱尔兰、欧盟、波罗的海、澳大利亚等各个国 家或区域开发的海洋生境分类方案及制图产品, 其 中部分国家/地区的海洋生境分类方案中包括了海 岸带分类, 在此对代表性的海洋生境分类和区划体 系进行归纳和介绍。

\subsection{1 全球海洋生境分区图}

全球性的海洋生境制图案例以“世界海洋生态 区系统” (Marine Ecoregions of the World, MEOW)和 “全球大洋和深海海底生物地理分类” (Global Open Oceans and Deep Seabed: Biogeographic Classification, GOODS)为代表, 分别对全球的近岸和深海 进行了全面的分区绘制。

2007年, 大自然保护协会(The Nature Conservancy, TNC)和世界自然基金会(World Wildlife Fund, WWF) 完善并提出了全球的海洋生态分区, 以提供 一个全面的近岸(至水深200 m)世界海洋生态区系 统(Spalding et al, 2007)。为保证该系统兼容已有的 国家或地区分类体系, 其组织架构采用了网络层次 方法, 以便于开展多尺度分析。在该系统内, 全球 海岸带和大陆架水域被划分成由 12 个生物带 (realm)、62个生物省 (province)和232个生态区 (ecoregion)构成的网络系统。其主要分区依据为海 岸带和大陆架水域的底栖及水层生物, 分区信息来 源包括期刊杂志、非政府组织报告、政府出版物等。 生物带分区的驱动因子包括水温、大时间和空间尺 度的地理隔离以及底栖生物亲缘关系; 生物省分区 的驱动因子包括地形特征(孤立的岛屿、大陆架系 统、半封闭海洋)、水文学特征(海流、上升流、海 
表1 全球海洋生境分区研究及方法总结(修改自Zhao \& Costello, 2019)

Table 1 Global marine habitat classifications and methods (Modified from Zhao \& Costello, 2019)

\begin{tabular}{|c|c|c|c|}
\hline 产品 Product & 方法 Methodology & 采用的数据和信息 Utilized data and information & 来源 Sources \\
\hline $\begin{array}{l}\text { 大洋生物地理边界图 Pelagic } \\
\text { biogeography boundaries }\end{array}$ & 专家意见 Expert opinion & 文献资料 Previous literature & van der Spoel, 1994 \\
\hline $\begin{array}{l}\text { 大海洋生态系内的渔业区划 } \\
\text { Fishery regions in Large Marine } \\
\text { Ecosystems }\end{array}$ & $\begin{array}{l}\text { 现有文献整合 Synthesizing } \\
\text { previous work }\end{array}$ & $\begin{array}{l}\text { 大海洋生态系和出版物 Large Marine } \\
\text { Ecosystem and publications }\end{array}$ & Watson et al, 2003 \\
\hline $\begin{array}{l}\text { 大洋表层生态分区(不包括海岸带) } \\
\text { Surface pelagic zones, not coastal }\end{array}$ & 专家意见 Expert opinion & $\begin{array}{l}\text { 海洋水色和理化数据 Ocean color and } \\
\text { physio-chemical data }\end{array}$ & Longhurst, 2007 \\
\hline $\begin{array}{l}\text { 世界海洋生态区系统 Coastal } \\
\text { marine ecoregions of the world }\end{array}$ & 专家意见 Expert opinion & $\begin{array}{l}\text { 已有的各区域的海岸带分区的综合资料 } \\
\text { Previous regional coastal mapping }\end{array}$ & Spalding et al, 2007 \\
\hline $\begin{array}{l}\text { 基于叶绿素 } a \text { 的海洋中上层生物群 } \\
\text { 落分类分区 Classification of }\end{array}$ & $\begin{array}{l}\text { 层次聚类分析 Hierarchical cluster } \\
\text { analysis }\end{array}$ & $\begin{array}{l}\text { 月均叶绿素 } a \text { Mean monthly chlorophyll- } a \\
\text { content }\end{array}$ & $\begin{array}{l}\text { Hardman-Mountford } \\
\text { et al, } 2008\end{array}$ \\
\hline
\end{tabular}

biomes based on chlorophyll- $a$ in pelagic ocean

全球海洋生物地理省分区 Global ocean biogeographic provinces

多聚类分析 Multivariate cluster analysis

专家意见 Expert opinion

全球大洋和深海海底分区 Global open oceans and deep seabed

classification

为全球公海海洋保护区设计的海洋 多元分析 Multivariate analysis

底栖生境图 Global benthic

seascapes for high seas marine

protected areas

全球深海(> $800 \mathrm{~m})$ 生物省分区

Biogeographic provinces for deep ocean floor (> $800 \mathrm{~m})$

大洋浮游生物多样性热点区

Oceanic phytoplankton hotspot regions

大洋生态区 Ecoregions of ocean

海洋动植物的生物地理带分区 Biogeographic realms of marine animals and flora

海洋水体的三维生态单元分区 Three-dimensional ecological marine clustering analysis

全球大洋中层生物地球化学分区
Global mesopelagic biogeochemical
provinces
世界海洋生态系统聚类分区
Clustered marine ecosystems of the
world ocean
专家意见 Expert opinion

Shannon熵值法 Shannon entropy

表层海流分带 Sea surface currents classification

物种出现数据的标准化聚类分析 Standardized cluster analysis of species occurrence data

$\mathrm{K}$-均值聚类分析 K-means

多元聚类分析和边界频次 Multivariate cluster analysis and boundary frequency

主成分分析和K-均值聚类分析

Principal component analysis and $\mathrm{K}$-means clustering analysis
MODIS/Aqua衍生的海表温度和海洋水色数据

Oliver \& Irwin, 2008 Sea surface temperature and ocean color from MODIS/Aqua reprocessing

现有研究的综述 Review of previous studies
水深、斜坡、初级生产力、沉积物厚度、底层 Harris \& Whiteway, 温度、底层溶解氧 Depth, slope, net primary 2009 productivity, sediment thickness, bottom temperature, and bottom dissolved oxygen

指示深海生物分布的理化变量 Physical and Watling et al, 2013 chemical variables indicating organism distribution at the deep-sea floor

海洋水色遥感 Remote sensing of ocean color Silvia et al, 2013

海流, 包括水温、盐度和上升流 Ocean currents, Bailey, 2014 including temperature, salinity, and upwellings

中上层和底栖生物分布数据 Pelagic and benthic Costello et al, 2017 species distribution data

水温、盐度、溶解氧、硝酸盐、磷酸盐和硅酸 Sayre et al, 2017 盐的三维环境变量 Three-dimensional environmental variables of temperature, salinity, oxygen, nitrate, phosphate, and silicate

世界大洋地图的中层环境数据 Mesopelagic Reygondeau et al, environmental data from World Ocean Atlas 2018

20项海洋环境变量 20 environmental variables Zhao et al, 2020
冰)及地球化学影响(大尺度的营养盐浓度和盐度); 生态区分区的驱动因子主要为局部地区占优势的 生物地理胁迫因子, 包括隔离程度、上升流、营养 盐输入、淡水通量、水温、海冰、沉积物、海流和 水深等。目前, 已有部分国际生物保护组织(如, TNC 和 WWF) 把MEOW系统划定的海洋生态区作 为保护管理工作的规划单元, 一些极具影响力的研
究成果也已采纳MEOW分区用于全球海洋保护区 的保护成效评估、人类活动对世界海洋的影响评 估、典型海洋生态系统的风险评估和管理对策研究 等(Beck et al, 2011; Edgar et al, 2014; Halpern et al, 2015)。

2009年, 联合国科教文组织出版了《全球大洋 和深海海底生物地理分类》报告, 首次尝试将全球 
的开阔大洋和深海海底划分为不同的生物地理区 域。此分类是由多学科专家组提出的, 报告将世界 海洋细分为 30 个生物省的水体分区, 跨越 3 大深度 (800-3,500 m, 3,500-6,500 m, >6,500 m)38个生物省 (14 个次深海区、14个深渊区和 10 个超深渊区)的底 栖生物分区, 以及10个热液喷口区。报告指出, 这 套生物分区方案旨在利用底层和水体环境的地球 物理和环境特征来识别具有相似生境和生物群落 特征的同质化区域, 其前提在于对生物分布和环境 关系的假设(UNESCO, 2009)。为提高其准确性, 未 来对海洋生物地理分类方案的改进还需依赖生物 等各方面信息资料的继续补充，这些资料最终可为 描述代表性海洋动植物区系的全球格局提供基础, 从而帮助人类了解海洋物种和栖息地的分布, 以便 进行科学的研究、保护和管理(Watling et al, 2013)。

\subsection{2 国家海洋生境制图方案}

从21世纪初开始, 美国、加拿大、澳大利亚、 英国和爱尔兰等国相继提出了国家层面的海洋生 境分类制图方案, 根据各国的海洋生态系统特点, 它们的分类体系和制图应用各有特色:

(1)美国海岸/海洋生态分类标准(Coastal and Marine Ecological Classification Standard, CMECS) (FGDC, 2012)。在21世纪初, 美国国家海洋和大气 管理局曾提出两个河口海洋生境分类系统, 以满足 自然资源管理者和科学家对识别和管理海洋生态 系统需要的统一分类标准和语言(Allee et al, 2000; Madden \& Grossman, 2004)。此后, 在上述标准基础 上修订形成了目前美国所采用的海岸/海洋生态分 类标准。其框架由水域环境和生物地理环境两大基 本环境背景(settings)构成, 包括水体、地形地貌、 底质、生物四个具体组分(components), 组合形成若 干个嵌套级别的分类。CMECS框架的设计适用于各 种空间尺度, 并可用于河口、海岸和开阔大洋系统 的沿海、底栖和中上层分区。在CMECS框架中还提 出了修饰因子概念(modifier), 该因子本身位于分类 层次之外, 但能够提供各分类的其他相关信息, 一 般包括底质、水质特征、物理属性、生物学属性等。 修饰因子在生境单元和生态小区的水平上十分重 要, 例如通常使用底质作为修饰因子来定义生境单 元, 而描述植被或群落的空间分布斑块/密度的修 饰因子是区分生态小区的重要特征。目前该分类标 准已用于美国多个河口海湾区域的底栖生物栖息
地分布图绘制、生态系统服务和生态恢复评估等研 究(Keefer et al, 2008; Carollo et al, 2013; Mittermayr et al, 2020)。

(2)加拿大海洋生境分类。加拿大为海洋保护规 划提出了一套海洋生境分类体系, 研究范围包括北 极、大西洋和太平洋区域截然不同的环境, 其目的 在于以统一的分类识别区域内代表性的或独特的 生境类型并划定其边界, 这是确定保护区位置和大 小的基本前提。该体系基于5个分类级别, 分别代表 海洋环境的 “持续”和周期性反复出现的地球物理特 征(Roff \& Taylor, 2000)。在该体系中, 其出发点认 为海洋群落的边界比陆地生态系统更具有动态性, 但仍有可能根据相对的“持续特征”来定义代表性群 落, 这些“持续特征” 的持续尺度大约在数百到数千 年的时间。由于加拿大地理范围的特点, 其生境分 类因子除了常见的海洋生物环境因子外, 还包括海 冰覆盖率这一独特因子。该体系提出, 由于许多区 域事实上缺乏海洋生物数据, 因此使用地球物理特 征替代海洋群落的方法应被广泛采纳, 并且在某些 情况下可能是唯一可行的方法, 而使用混合物理和 生物学特征(例如指示种)反而可能是一些分类方案 的弱点。

(3)澳大利亚生境分类数据库。澳大利亚早期使 用澳大利亚海洋环境生物分区表进行生境分类, 其 将环境分为大的单元 $\left(3,000-240,000 \mathrm{~km}^{2}\right)$, 这些单 元具有广泛的自然特征和影响整个生态系统功能 的环境过程(IMCRA，1998)。但IMCRA的空间分辨 率过粗, 无法定义栖息地, 也无法发现群落的变化 或损失。其后澳大利亚国家数据服务(Australian National Data Service)基于更高分辨率提出了澳大 利亚海底生境分类方案, 并建立了“澳大利亚海洋 地图” (SEAMAP) 空间数据库。其中包括生物地理和 水域两个维度, 以及水体、地貌、底质、生物四个 组分(Butler et al, 2017), 这与美国和欧盟的生境分 类体系具有相似之处, SEAMAP将澳大利亚近岸栖 息地分为37类25,684个斑块。

(4)英国和爱尔兰海洋生境分类。联合自然保护 委员会(Joint Nature Conservation Committee, JNCC) 开发了一种海洋生境分类系统The Marine Habitat Classification for Britain and Ireland, 作为管理与保 护英国和爱尔兰海洋栖息地的辅助工具(Connor et al, 2004)。该分类系统包括6个级别, 其顶层分类区 
分为海洋、陆地和淡水系统, 在高级层次中识别了 影响生物群落结构的各种环境因素, 包括底质、水 层、暴露性、海洋学、水质和其他物理参数。其中 水平梯度的底质和垂直梯度的水层似乎是决定群 落分布的主要因素, 这些也是最易记录和最可靠的 属性, 因此, 底质和水层被认为适于构建较高级别 的分类。而在较低级别的层次上, 由群落生境和亚 群落生境来定义(Parry, 2019)。通过对调查数据进行 统计分析, 将数据分组为具有相似特征的聚类, 这 是定义群落生境和亚群落生境的基础。群落生境由 群落中存在和丰度均最“显著”的物种组合决定。亚 群落生境是生物群落的进一步分类, 并且使用相对 不那么显著的物种来定义。通常在制图时, 群落生 境的规模至少为 $25 \mathrm{~m}^{2}$, 大尺度上可覆盖若干平方 公里。

\subsection{3 区域海洋生境制图方案}

区域性的海洋生境分类制图目前主要以欧洲 地区为代表, 其中包括一个泛欧洲区域的生境分类 体系, 以及以北欧海洋生境为特色的波罗的海生物 群落和生境分类系统。

欧洲自然信息系统 (the European Nature Information System, EUNIS) 是由欧洲环境署开发的 泛欧洲生境分类体系(Moss, 2008; Evans, 2012; Evans et al, 2014), 其地理范围为欧洲大陆外的海 洋, 包括离岸岛屿以及欧盟成员国的群岛。EUNIS 的海洋计划涵盖了从潮间带到深渊的整个海床, 还 包括一部分中上层生境。整个EUNIS生境大类大约 有 30 个, 其下包括若干小类, 能支持对 $1 \mathrm{~m}^{2}$ 至 100 $\mathrm{m}^{2}$ 的小尺度样本进行分类。近海的一些生境则被归 类为“生境综合体”, 涵盖生境的常见组合或镶嵌体, 通常占地至少 10 ha, 例如包括潮间带、滩涂、盐沼 和其他沿海生境的河口。EUNIS生境分类方案与美 国CMECS标准的不同之处在于, EUNIS方案描述的 生境类型以单一层次排列(而非互相嵌套), 形成了 海洋生境分类的 6 个级别。较高的级别关注物理特 征, 而较低的级别描述生境的生物成分。目前, 该 体系下已形成了泛欧洲的大尺度EUNIS海洋生境 地图 (EU SeaMap) (Cameron \& Askew, 2011; Vasquez et al, 2015; Populus et al, 2017)以及其他各 类地方生境地图(Bekkby et al, 2017), 并实现了各 尺度生境地图的在线发布和查询, 是各个国家/地 区的生境制图方案里数据应用产品最丰富的。
波罗的海生物群落和生境分类系统(HELCOM HUB) 是波罗的海海洋环境保护委员会 (Baltic Marine Environment Protection Commission-Helsinki Commission, HELCOM) 为波罗的海海洋生物群落 生境开发的综合分类系统(HELCOM, 2013a)。它起 源于 HELCOM ECNATURE 红色清单项目 (HELCOM，1998), 这是第一个基于底质类型和水 深的波罗的海通用生境分类方案, 其分类规则主要 依靠专家判断, 但不包括生物学分类标准。在2007 年, HELCOM红色名录生物群落专家小组设定了更 新红色名录分类系统的目标, 从而将生物分类加入 该方案。该项研究曾尝试直接将EUNIS应用于波罗 的海地区, 但发现该方案难以指示波罗的海本地海 洋生境的生物和非生物特征 (Galparsoro et al, 2012)。目前的HELCOM HUB与EUNIS兼容, 并兼 顾了波罗的海特有的海洋生物群落信息。HELCOM HUB主要关注底栖生境/生物群落, 仅在分类系统 的较高层次考虑水层环境, 其中使用了光照穿透深 度作为主要变量, 这与传统的分类方案有所区别。 HELCOM HUB为非生物和生物学分类标准提供了 清晰的定量分类规则，目前该体系已被波罗的海国 家广泛使用，例如被用作制定德国“北海和波罗的 海受威胁栖息地类型红色名录” 国家分类系统的基 础(Finck et al, 2017), 并被若干国家尺度的案例研 究采纳(Schiele et al, 2014, 2015)。此外, HELCOM HUB还可以作为依据用于划分并评估海洋和沿海 红色名录生物群落及生物群落复合体(HELCOM, 2013b)。

\subsection{4 小结}

综上所述, 各个国家和研究机构目前提出的生 境制图方案各有特色, 说明任何用于生境制图的方 法都取决于各主导团体的研究与管理目标。综合现 有各项研究中的分类分区方法, 我们总结了海洋生 境的共性分异特征: 海洋生境的基本分异规律包括 水平梯度的底层分异、垂直梯度的水层分异和其他 因子引起的局部或微域分异。水层分异决定了河 口、海岸、大洋、深海等系统的分布, 而底层分异 决定了生物群落生境的水平分布范围。水平梯度和 垂直梯度的分异构成了海洋生境在三维空间上的 多样性, 也是现有通行的多数海洋生境分类方案内 具备的两大背景支柱。在这两大支柱的框架下, 各 分类方案根据管理目标的差异选择不同的环境/生 
物驱动因子, 构成层级化或嵌套的分类方案并制定 分区。

海洋生境分类方案是海洋生境制图的核心, 虽 然在制定具体生境分类方案时应当因地制宜，但为 了保证方案的易用性和可操作性, 制定过程中仍应 遵循一定的通用规则，本文在总结全球、国家和区 域海洋生境制图案例的基础上对海洋生境分类方 案应具备的特征进行了归纳(表2)。

\section{2 国内研究进展}

自20世纪50年代以来, 我国开展了多次大规模 的全国性海洋专项调查，包括1958-1960年开展的 全国海洋综合调查、20世纪80年代开展的全国海岸 带和海涂资源综合调查、20世纪90年代开展的中国 海岛资源综合调查、2000年后开展的我国近海海洋 综合调查与评价等, 调查内容包括海洋水文、海洋
气象、海洋化学、海洋生物、海洋地质和社会经济 等多项要素，通过这些调查获取了宝贵的海洋观测 数据记录, 形成了相关的图集、图志, 初步掌握了 中国近海环境和生物要素的基本特征和变化规律。 2010年后随着海洋科研调查水平的提升，中国海洋 调查的范围更是向大洋扩展。但以往的调查和图集 成果仍然是以航次、学科为主题进行收集和产出, 缺乏成熟的标准化生境制图方案将这些不同来源、 不同形式的观测数据进行系统整合和分区分类绘 制, 从而科学确定海洋生态单元边界、形成现代化 的海洋生境地图。

国内此前海域空间管理的主要依据是海洋功 能区划，主要以用海需求界定空间分区，而近年来 我国的海洋国土空间管理理念逐渐向基于生态系 统的管理转变, 开始以海洋生态系统的基本类型及

\section{表2 海洋生境分类方案特征列表(修改自Ball et al, 2006)}

Table 2 Characteristics of marine habitat classification (Modified from Ball et al, 2006)

\begin{tabular}{ll}
\hline $\begin{array}{l}\text { 序号 } \\
\text { Number }\end{array}$ & 特征名称 Name \\
\hline 1 & 分级化 Hierarchical \\
2 & 互斥性 Exclusive \\
3 & 全面性 Comprehensive \\
4 & $\begin{array}{l}\text { 可识别性和重复性 } \\
\text { Identifiable and repeatable }\end{array}$ \\
5 & 可描述性 Descriptive
\end{tabular}

特征描述 Description

分类系统应该是分级的，以避免定义的重叠以及在系统不同层次上出现类别重复，并确保在生态上相似 的类型彼此接近并处于恰当的级别。The classification system should be hierarchical to avoid overlap of definitions and duplication of categories at different levels of the system, and ensure that ecologically similar types are placed near to each other and at an appropriate level.

分类方案应互斥且详尽, 每个要分类的要素都应仅属于一个类。A classification scheme should be mutually exclusive and exhaustive so that every feature to be classified should fall within one class only.

全面考虑制图区域内的所有海洋生境类型。Be comprehensive, accounting for all the marine habitats within the mapping region.

生境应该是可识别的、有重复性的环境单元类型。Habitats should be identifiable, repeatable environmental units.

6 易用性 Practical

可提供通用且易于理解的语言来描述海洋生境。Provide a common and easily understood language for the description of marine habitats.

7 通用性 Versatility 形式实用，表述清晰。Be practical in format and clear in its presentation.

保证各种生境采样技术都能产生相同的生境类别或群落定义, 尽管生境分类分区的等级可能取决于采 样技术的分辨率。All types of sampling techniques should result in the same habitat classes or community definitions, although the level to which a habitat can be classified in a hierarchy will be dependent on the resolution of the sampling technique.

8 动态性 Dynamic 分类体系应能识别动态的时间尺度。与在较长时间范围内变化的生境因子(例如礁岩)相比, 在较短时间 范围内变化的生境因子(例如生物群落)应纳入在层次分类体系中的较低级别。The classification should recognize time scales over which variables may change. Habitat variables that change over shorter time scales (e.g. biota) should be incorporated at a lower level in the hierarchy than variables that change over longer time scales (e.g. reef substratum).

9 普遍性 Universality

它应包括足够的细节, 以供自然资源管理人员和监测人员应用; 但也应足够宽泛 (通过层次结构), 以使 宏观的生境信息能够在国家和全球层面上呈现或供非专业人员使用。It should include sufficient detail to be of practical use for resource managers and field surveyors, but be sufficiently broad (through hierarchical structuring) to enable summary habitat information to be presented at national and international levels or be used by nonspecialists.

10 可扩展性 Scalable 它应该足够灵活, 能够通过添加新信息进行修改, 但又要足够稳定以支持正在进行的应用。修改的内容 可以被清晰地记录, 有可能的话可将新定义的类型重新关联到之前的分类体系中。It should be sufficiently flexible to enable modification resulting from the addition of new information, but stable enough to support ongoing uses. Changes should be clearly documented and where possible, newly defined types need to be related back to types in earlier versions of the classification. 
其特征引导空间规划, 例如海洋生态保护红线(胡 文佳等, 2020)。在实践过程中发现, 现有规划普遍 缺乏充分的海洋生物地理空间数据支持, 存在家底 不明、空间边界难以确定等问题。以往也有一些学 者尝试探索综合性的海洋生态分区绘制方案, 例如 王传胜等(2014)在生态重要性和环境胁迫性评价的 基础上将辽宁海岸带划分成4类重要生态空间; Huang等(2015)在中国近海开展了海洋生物多样性 保护优先区的研究, 按生物地理因素将中国近海划 分为98个分区, 其中有 43 个分区被确定为保护优先 区; 陈甘霖等(2017)对福建东山县海洋生态系统分 类和划分, 识别了红树林生态系统、珊瑚礁生态系 统、海岛生态系统、渔业资源的育幼场和珍稀濒危 物种分布区等。但总体来看, 国内尚缺乏真正意义 上的海洋生境制图研究, 对海洋生态分类分区综合 体系的探索仍处于起步阶段, 分类依据仍以传统的 海洋生态系统类型或生物保护等级为主, 尚未满足 跨学科、跨专业的自然资源综合管理需求。

在中央强化国土空间规划体系、推进生态系统 保护和修复等战略意见的指导下, 海洋生态空间和 生产空间的统筹规划是未来海洋和海岸带国土空 间管理工作的基石。随着我国对于海洋生态空间管 理的升级, 亟待将生物群落和生态系统与海洋生物 地理环境相匹配, 形成定量化、精细化的海洋生境 制图, 从而准确识别海洋生态空间的管控边界; 此 外, 出于我国海洋开发战略的需求, 亟待通过海洋 生境制图的研究为海洋生产空间选址布局提供依 据, 并根据不同海域/海区的生境类型设计相应的 受损生境修复和渔业资源恢复方案(杨红生等, 2019), 探索海洋生物资源可持续利用的方式。

\section{4 总结与展望}

海洋生境制图是海洋生态系统空间管理的基 础, 旨在探寻海洋环境与生物群落/物种的分布规 律, 为生物多样性保护和资源空间管理工作提供可 以依循的边界。海洋生境分类方案的具体内容与研 究的目标和空间尺度相关, 总体来看“以不变应万 变” 是主流趋势, 即优先采用变化少的分类指标以 及周期变化的分类指标, 以保证分类区划体系的稳 定性、完整性和科学性。海洋生境分区的划分方法 大致可分为定性和定量两种。专家咨询法是定性分 析的典型代表, 目前已有多个权威性的区划方案是
在文献资料的基础上采用专家咨询法确定的。而对 于定量分区方法, 多元统计和聚类分析是较多被采 用的，其中K-均值聚类分析在近年来发表的多个分 区案例研究中得到了有效的应用。目前已有多个国 家和研究机构提出了全球和区域尺度的海洋生境 制图方案，并在相关的海洋保护区规划、濒危物种 栖息地名录制定、海洋生境变化监测和海洋空间规 划等管理活动中发挥了重要的作用。

我国海洋国土从北至南穿过温带、亚热带和热 带3个气候带, 覆盖黄海、东海、黑潮暖流、南中国 海等大海洋生态系, 大陆海岸线超过 $18,000 \mathrm{~km}$, 岛 屿岸线 $14,000 \mathrm{~km}$, 拥有近 300 万 $\mathrm{km}^{2}$ 的主张管辖海 域(王永生, 2004), 以及丰富的海洋生物多样性。探 索海洋生境的空间分布规律, 了解海区内生态系统 和生物群落特征与物理化学环境因子之间的关系, 科学划分海洋生物多样性保护与海洋国土生态空 间的管理边界, 对于推进基于生态系统的海洋管 理、实现海洋生态文明和可持续发展目标具备关键 的意义。基于国内外目前的研究进展, 笔者认为, 未来国内亟待开展以下几个方面的研究:

(1)研究制定中国特色海洋生境分类方案。在国 外各海洋生境分类方案的基础上继续深入理论方 法的探讨, 构建起符合中国海洋生态系统特色的海 洋生境分类方案, 完善、深化对我国海洋生境及其 地域分异规律的认知。

(2)海洋生境区划技术研究。开展海洋生物地理 分区的研究和实践, 将分类方案、分区方法和生态 过程进行有机结合, 分区过程中既关注海洋生态系 统的完整性，又兼顾海洋生态系统的连通性，既将 分区单元作为生物与环境的相对独立整体来认识, 又重视生态过程的联系。

(3)海洋生境基础信息融合和数据库建立。灵活 融合现场采样、卫星遥感、船载测量等各种数据调 查成果，基于最新的空间分析技术，建立不同区域 尺度的生物/环境信息数据库, 以便进行存储、检索 和分区, 并据此开展各尺度下不同分区单元的图文 特征描述。

(4)形成决策辅助用地图数据产品。为了支持海 洋生物多样性保护和海洋国土生态空间管理等工 作, 应根据管理需求有针对性地生产各类海洋生境 制图产品, 一些重要的产品可能包括国家海洋生态 系统类型分布图、近岸和近海底栖生境制图、主要 
渔业资源产卵场和育幼场分布图等, 从而为我国海 洋生态保护红线划定调整、海洋空间规划修编、海 洋保护地网络建设、海洋牧场选址规划、海洋生态 保护与修复规划等行动提供关键科学依据。

\section{ORCID}

杜建国 (iD) https://orcid.org/0000-0002-8722-903X

\section{参考文献}

Allee RJ, Dethier M, Brown D, Deegan L, Ford RG, Hourigan TF, Maragos J, Schoch C, Sealey K, Twilley R, Weinstein MP, Yoklavich M (2000) Marine and Estuarine Ecosystem and Habitat Classification. NOAA/National Marine Fisheries Service, Silver Spring, MD.

Bailey RG (2014) Ecoregions: The Ecosystem Geography of the Oceans and Continents. Springer, New York.

Ball D, Blake S, Plummer A (2006) Review of Marine Habitat Classification Systems. Parks Victoria, Melbourne, Australia.

Beck MW, Brumbaugh RD, Airoldi L, Carranza A, Coen LD, Crawford C, Defeo O, Edgar GJ, Hancock B, Kay MC, Lenihan HS, Luckenbach MW, Toropova CL, Zhang GF, Guo XM (2011) Oyster reefs at risk and recommendations for conservation, restoration, and management. BioScience, 61, 107-116.

Bekkby T, Gerovasileiou V, Papadopoulou KN, Sevastou K, Dailianis T, Fiorentino D, McOwen C, Smith CJ, Amaro T, Bakran-Petricioli T, Bilan M, Boström C, Carreiro-Silva M, Carugati L, Cebrian E, Cerrano C, Christie H, Danovaro R, Eronat EGT, Fraschetti S, Gagnon K, Gambi C, Grehan A, Hereu B, Kipson S, Kizilkaya IT, Kotta J, Linares C, Milanese M, Morato T, Ojaveer H, Orav-Kotta H, Pham CK, Rinde E, Sarà A, Scharfe M, Scrimgeour R (2017) State of the Knowledge on European Marine Habitat Mapping and Degraded Habitats. http://www.merces-project.eu/sites/def ault/files/MERCES_D1.1_0.pdf. (accessed on 2020-03-01)

Bland LM, Keith DA, Miller RM, Murray NJ, Rodríguez JP (2017) Guidelines for the Application of IUCN Red List of Ecosystems Categories and Criteria, Version 1.1. IUCN, Gland, Switzerlands.

Boswarva K, Butters A, Fox CJ, Howe JA, Narayanaswamy B (2018) Improving marine habitat mapping using high-resolution acoustic data; a predictive habitat map for the Firth of Lorn, Scotland. Continental Shelf Research, 168, 39-47.

Brown CJ, Smith SJ, Lawton P, Anderson JT (2011) Benthic habitat mapping: A review of progress towards improved understanding of the spatial ecology of the seafloor using acoustic techniques. Estuarine, Coastal and Shelf Science, 92, 502-520.

Buhl-Mortensen L, Buhl-Mortensen P, Dolan MJF, GonzalezMirelis G (2015a) Habitat mapping as a tool for conservation and sustainable use of marine resources: Some perspectives from the MAREANO Programme, Norway. Journal of Sea Research, 100, 46-61.

Buhl-Mortensen L, Buhl-Mortensen P, Dolan MFJ, Holte B (2015b) The MAREANO programme-A full coverage mapping of the Norwegian off-shore benthic environment and fauna. Marine Biology Research, 11, 4-17.

Butler C, Lucieer V, Walsh P, Flukes E, Johnson C (2017) Seamap Australia [Version 1.0] the Development of a National Benthic Marine Classification Scheme for the Australian Continental Shelf. The Institute for Marine and Antarctic Studies, University of Tasmania.

Cameron A, Askew N (2011) EUSeaMap-Preparatory Action for Development and Assessment of a European Broad-Scale Seabed Habitat Map. https://webgate.ec.europa. eu/maritimeforum/system/files/20110301_FinalReport_EUS eaMap_v2.9.pdf. (accessed on 2020-03-01)

Carey DA, Hayn M, Germano JD, Little DI, Bullimore B (2015) Marine habitat mapping of the Milford Haven Waterway, Wales, UK: Comparison of facies mapping and EUNIS classification for monitoring sediment habitats in an industrialized estuary. Journal of Sea Research, 100, 99-119.

Carollo C, Allee RJ, Yoskowitz DW (2013) Linking the Coastal and Marine Ecological Classification Standard (CMECS) to ecosystem services: An application to the US Gulf of Mexico. International Journal of Biodiversity Science, Ecosystem Services \& Management, 9, 249-256.

Chen GL, Hu WJ, Chen B, Ma ZY, Liao JJ (2017) Marine spatial planning using in marine ecological red line zoning-A case study of Dongshan County. Journal of Applied Oceanography, 36, 6-15. (in Chinese with English abstract) [陈甘霖，胡文佳，陈涁，马致远，廖建基 (2017) 海洋空间规划技术在小尺度海洋生态红线区划中的应用 一以东山县海域为例. 应用海洋学学报, 36, 6-15.]

Cogan CB, Todd BJ, Lawton P, Noji TT (2009) The role of marine habitat mapping in ecosystem-based management. ICES Journal of Marine Science, 66, 2033-2042.

Connor DW, Allen JH, Golding N, Howell KL, Lieberknecht LM, Northen KO, Reker JB (2004) The Marine Habitat Classification for Britain and Ireland, Version 04.05. Joint Nature Conservation Committee, Peterborough, UK.

Costello MJ (2009) Distinguishing marine habitat classification concepts for ecological data management. Marine Ecology Progress Series, 397, 253-268.

Costello MJ, Tsai P, Wong PS, Cheung AKL, Basher Z, Chaudhary C (2017) Marine biogeographic realms and species endemicity. Nature Communications, 8, 1057.

Cropper A (1993) Convention on Biological Diversity. Environmental Conservation, 20, 364.

Crowder L, Norse E (2008) Essential ecological insights for marine ecosystem-based management and marine spatial planning. Marine Policy, 32, 772-778. 
de la Hoz CF, Ramos E, Puente A, Méndez F, Menéndez M, Juanes JA, Losada ÍJ (2018) Ecological typologies of large areas: An application in the Mediterranean Sea. Journal of Environmental Management, 205, 59-72.

Diaz RJ, Solan M, Valente RM (2004) A review of approaches for classifying benthic habitats and evaluating habitat quality. Journal of Environmental Management, 73, 165-181.

European Commission (2007) Data Completeness, Quality and Coherence. Guidance document on "Habitats Directive", European Commission.

Edgar GJ, Stuart-Smith RD, Willis TJ, Kininmonth S, Baker SC, Banks S, Barrett NS, Becerro MA, Bernard ATF, Berkhout J, Buxton CD, Campbell SJ, Cooper AT, Davey M, Edgar SC, Försterra G, Galván DE, Irigoyen AJ, Kushner DJ, Moura R, Parnell PE, Shears NT, Soler G, Strain EMA, Thomson RJ (2014) Global conservation outcomes depend on marine protected areas with five key features. Nature, 506, 216-220.

Evans D (2012) The EUNIS habitats classification: Past, present and future. Revista de Investigación Marina, 19, 28-29.

Evans D, Condé S, Gelabert ER (2014) Crosswalks between European Marine Habitat Typologies-A Contribution to the MAES Marine Pilot. ETC/BD Report for the EEA.

FGDC (2012) Coastal and Marine Ecological Classification Standard. FGDC-STD-018-2012, United States.

Finck P, Heinze S, Raths U, Riecken U, Ssymank A (2017) Red List of Endangered Biotope Types in Germany-Third Updated Version in 2017. The Federal Agency of Nature Conservation, Berlin.

Fontaine A, Devillers R, Peres-Neto PR, Johnson LE (2015) Delineating marine ecological units: A novel approach for deciding which taxonomic group to use and which taxonomic resolution to choose. Diversity and Distributions, 21, 1167-1180.

Gagne TO, Reygondeau G, Jenkins CN, Sexton JO, Bograd SJ, Hazen EL, Van Houtan KS (2020) Towards a global understanding of the drivers of marine and terrestrial biodiversity. PLoS ONE, 15, 0228065.

Galparsoro I, Connor DW, Borja A, Aish A, Amorim P, Bajjouk T, Chambers C, Coggan R, Dirberg G, Ellwood H, Evans D, Goodin KL, Grehan A, Haldin J, Howell K, Jenkins C, Michez N, Mo G, Buhl-Mortensen P, Pearce B, Populus J, Salomidi M, Sánchez F, Serrano A, Shumchenia E, Tempera F, Vasquez M (2012) Using EUNIS habitat classification for benthic mapping in European seas: Present concerns and future needs. Marine Pollution Bulletin, 64, 2630-2638.

Grant S, Constable A, Raymond B, Doust S (2006) Bioregionalisation of the Southern Ocean. CE-CRC and WWF, Australia.

Gray JS (1997) Marine biodiversity: Patterns, threats and conservation needs. Biodiversity and Conservation, 6, 153-175.

Halpern BS, Frazier M, Potapenko J, Casey KS, Koenig K, Longo C, Lowndes JS, Rockwood RC, Selig ER, Selkoe KA, Walbridge S (2015) Spatial and temporal changes in cumulative human impacts on the world's ocean. Nature Communications, 6, 7615.

Hardman-Mountford NJ, Hirata T, Richardson KA, Aiken J (2008) An objective methodology for the classification of ecological pattern into biomes and provinces for the pelagic ocean. Remote Sensing of Environment, 112, 3341-3352.

Harris PT, Baker EK (2020) Why map benthic habitats? Seafloor geomorphology as benthic habitat. In: Seafloor Geomorphology as Benthic Habitat (eds Harris PT, Baker EK), pp. 3-22. Elsevier Science.

Harris PT, Whiteway T (2009) High seas marine protected areas: Benthic environmental conservation priorities from a GIS analysis of global ocean biophysical data. Ocean and Coastal Management, 52, 22-38.

HELCOM (1998) Red List of Marine and Coastal Biotopes and Biotopes Complexes of the Baltic Sea, Belt Sea and Kattegat. Helsinki Commission, Helsinki, Finland.

HELCOM (2013a) HELCOM HUB-Technical Report on the HELCOM Underwater Biotope and Habitat Classification. Helsinki Commission, Helsinki, Finland.

HELCOM (2013b) Red List of Baltic Sea Underwater Biotopes, Habitats and Biotope Complexes. Helsinki Commission, Helsinki, Finland.

Hu WJ, Chen B, Ma ZY, Yu WW, Xiao JM, Zhang D (2020) The research progress and enlightenment of ecosystem-based marine spatial planning. Ocean Development and Management, 37(4), 3-11. (in Chinese with English abstract) [胡文佳, 陈涁, 马志远, 俞炜炜, 肖 佳媚, 张典 (2020) 基于生态系统的海洋空间规划: 研究 进展与启示. 海洋开发与管理, 37(4), 3-11.]

Huang H, Chen B, Lin JL (2015) The marine spatial classification and the identification of priority conservation areas (PCAs) for marine biodiversity conservation-A case study of the offshore China. Ocean and Coastal Management, 116, 224-236.

Huang Z, Brooke BP, Harris PT (2011) A new approach to mapping marine benthic habitats using physical environmental data. Continental Shelf Research, 31, S4-S16.

ICES (2005) Report of the Working Group on Habitat Mapping (WGMHM), p. 87. ICES, Bremerhaven.

ICES (2008) ICES Science Plan, 2009-2013, p. 14. ICES, Copenhagen.

IMCRA (1998) Interim Marine and Coastal Regionalisation for Australia: An Ecosystem-based Classification for Marine and Coastal Environments, Version 3.3. IMCRA Technical Group, Canberra.

Katsanevakis S, Stelzenmüller V, Andy S, Sørensen TK, Jones 
PJS, Kerr S, Badalamenti F, Anagnostou C, Breen P, Chust G, D’Anna G, Duijn M, Filatova T, Fiorentino F, Hulsman H, Johnson K, Karageorgis AP, Kröncke I, Mirto S, Pipitone C, Portelli S, Qiu WF, Reiss H, Sakellariou D, Salomidi M, van Hoof L, Vassilopoulou V, Fernández TV, Vöge S, Weber A, Zenetos A, ter Hofstede R (2011) Ecosystem-based marine spatial management: Review of concepts, policies, tools, and critical issues. Ocean and Coastal Management, 54, 807-820.

Keefer ML, Peery CA, Wright N, Daigle WR, Caudill CC, Clabough TS, Griffith DW, Zacharias MA (2008) Evaluating the NOAA Coastal and Marine Ecological Classification Standard in estuarine systems: A Columbia River Estuary case study. Estuarine, Coastal and Shelf Science, 78, 89-106.

Livingstone TC, Harris JM, Lombard AT, Smit AJ, Schoeman DS (2018) Classification of marine bioregions on the east coast of South Africa. African Journal of Marine Science, 40, 51-65.

Longhurst A (2007) Ecological Geography of the Sea. Academic Press, Boston.

Madden CJ, Grossman DH (2004) Coastal/Marine Systems of North America: Framework for an Ecological Classification Standard. NatureServe, Arlington, Virginia.

Malcolm HA, Jordan A, Schultz AL, Smith SDA, Ingleton T, Foulsham E, Linklater M, Davies P, Ferrari R, Hill N, Lucieer V (2016) Integrating seafloor habitat mapping and fish assemblage patterns improves spatial management planning in a marine park. Journal of Coastal Research, 75, 1292-1296.

McGowan J, Hines E, Elliott M, Howar J, Dransfield A, Nur N, Jahncke J (2013) Using seabird habitat modeling to inform marine spatial planning in central California's National Marine Sanctuaries. PLoS ONE, 8, e71406.

McLeod KL, Lubchenco J, Palumbi SR, Rosenberg AA (2005) Scientific Consensus Statement on Marine Ecosystem-based Management, USA. https://marineplanning.org/wp-content/ uploads/2015/07/Consensusstatement.pdf. (accessed on 2020-03-21)

Mittermayr A, Legare B, Borrelli M (2020) Applications of the Coastal and Marine Ecological Classification Standard (CMECS) in a Partially Restored New England Salt Marsh Lagoon. Estuaries and Coasts, doi:10.1007/s12237-020 $-00707-2$.

Monte SD, Soccodato A, Alvain S, d'Ovidio F (2013) Can we detect oceanic biodiversity hotspots from space? The ISME Journal, 7, 2054.

Moss D (2008) EUNIS Habitat Classification-A Guide for Users. European Topic Centre on Biological Diversity.

Mumby PJ, Harborne AR (1999) Development of a systematic classification scheme of marine habitats to facilitate regional management and mapping of Caribbean coral reefs. Biological Conservation, 88, 155-163.
Oliver MJ, Irwin AJ (2008) Objective global ocean biogeographic provinces. Geophysical Research Letters, 35, L15601.

Parry MEV (2019) Guidance on Assigning Benthic Biotopes using EUNIS or the Marine Habitat Classification of Britain and Ireland. JNCC, Peterborough.

Populus J, Vasquez M, Albrecht J, Manca E, Agnesi S, Al Hamdani Z, Andersen J (2017) EUSeaMap, a European Broad-scale Seabed Habitat Map. https://doi.org/10.13155/ 49975. (accessed on 2020-03-21)

Ramos E, Puente A, Guinda X, Juanes JA (2017) A hierarchical ecological classification system along the NE Atlantic coast: Focusing on the local scale (Cantabria, N Spain). European Journal of Phycology, 52, 75-89.

Reygondeau G, Guidi L, Beaugrand G, Henson SA, Koubbi P, Mackenzie BR, Sutton TT, Fioroni M, Maury O (2018) Global biogeochemical provinces of the mesopelagic zone. Journal of Biogeography, 45, 500-514.

Robinson CLK, Levings CD (1995) An overview of habitat classification systems, ecological models, and geographic information systems applied to shallow foreshore marine habitats. British Journal of Criminology, 7, 3-10.

Roff JC, Taylor ME (2000) National frameworks for marine conservation-A hierarchical geophysical approach. Aquatic Conservation Marine and Freshwater Ecosystems, 10, 209-223.

Sayre RG, Wright DJ, Breyer SP, Butler KA, Van Graafeiland K, Costello MJ, Harris PT, Goodin KL, Guinotte JM, Basher Z, Kavanaugh MT, Halpin PN, Monaco ME, Cressie N, Aniello P, Frye CE, Stephens D (2017) A three-dimensional mapping of the ocean based on environmental data. Oceanography, 30, 90-103.

Schiele KS, Darr A, Zettler ML (2014) Verifying a biotope classification using benthic communities-An analysis towards the implementation of the European Marine Strategy Framework Directive. Marine Pollution Bulletin, 78, 181-189.

Schiele KS, Darr A, Zettler ML, Friedland R, Tauber F, von Weber M, Voss J (2015) Biotope map of the German Baltic Sea. Marine Pollution Bulletin, 96, 127-135.

Sotheran I, Benson A, Crawford-Avis O (2014) Mapping Habitats and Biotopes from Acoustic Datasets to Strengthen the Information Base of Marine Protected Areas in Scottish Waters. JNCC, Peterborough.

Spalding MD, Fox HE, Allen GR, Davidson N, Ferdaña ZA, Finlayson M, Halpern BS, Jorge MA, Lombana A, Lourie SA, Martin KD, McManus E, Molnar J, Recchia CA, Robertson J (2007) Marine ecoregions of the world: A bioregionalization of coastal and shelf areas. BioScience, 57, 573-583.

Stevens T, Connolly RM (2004) Testing the utility of abiotic surrogates for marine habitat mapping at scales relevant to management. Biological Conservation, 119, 351-362. 
Strong JA, Clements A, Lillis H, Galparsoro I, Bildstein T, Pesch R (2019) A review of the influence of marine habitat classification schemes on mapping studies: Inherent assumptions, influence on end products, and suggestions for future developments. ICES Journal of Marine Science, 76, 10-22.

TFMPA ANZECC (Task Force on Marine Protected Areas of Australian and New Zealand Environment and Conservation Council) (2000) NRSMPA Strategic Plan of Action: Review of Methods for Ecosystem Mapping for the NRSMPA, Report on Action 8. Connell Wagner for Environment, Australia.

Thiault L, Weekers D, Curnock M, Marshall N, Pert PL, Beeden R, Dyer M, Claudet J (2020) Predicting poaching risk in marine protected areas for improved patrol efficiency. Journal of Environmental Management, 254, 109808.

UNESCO (2009) Global Open Oceans and Deep Seabed (GOODS) Biogeographic Classification. UNESCO-IOC, Paris.

van der Spoel S (1994) The basis for boundaries in pelagic biogeography. Progress in Oceanography, 34, 121-133.

Vasquez M, Chacón DM, Tempera F, O’Keeffe E, Galparsoro I, Alonso JLS, Gonçalves JMS, Bentes L, Amorim P, Henriques V, McGrath F, Monteiro P, Mendes B, Freitas R, Martins R, Populus J (2015) Broad-scale mapping of seafloor habitats in the north-east Atlantic using existing environmental data. Journal of Sea Research, 100, 120-132.

Vassallo P, Bianchi CN, Paoli C, Holon F, Navone A, Bavestrello G, Vietti RC, Morri C (2018) A predictive approach to benthic marine habitat mapping: Efficacy and management implications. Marine Pollution Bulletin, 131, 218-232.

Wang CS, Zhu SS, Dang LJ (2014) Key ecological spaces division of coastal Liaoning. Resources Science, 36, 1739-1747. (in Chinese with English abstract) [王传胜, 朱 珊珊, 党丽娟 (2014) 辽宁海岸带重点生态空间分类研
究. 资源科学, 36, 1739-1747.]

Wang YS (2004) WTO and marine resources development and management in China. Ocean Development and Management, 21(1), 11-15. (in Chinese) [王永生 (2004) WTO与我国海洋资源开发与管理. 海洋开发与管理, 21(1), 11-15.]

Ware S, Downie AL (2020) Challenges of habitat mapping to inform marine protected area (MPA) designation and monitoring: An operational perspective. Marine Policy, 111, 103717.

Watling L, Guinotte J, Clark MR, Smith CR (2013) A proposed biogeography of the deep ocean floor. Progress in Oceanography, 111, 91-112.

Watson R, Pauly D, Christensen V, Froese R, Longhurst A, Platt T, Sathyendranath S, Sherman K, O'reilly J, Celone P (2003) Mapping fisheries onto marine ecosystems for regional, oceanic and global integrations. In: Large Marine Ecosystems of the World: Trends in Exploitation, Protection, and Research (eds Hempel G, Sherman K), pp. 365-396. Elsevier, Amsterdam.

Wright DJ, Heyman WD (2008) Introduction to the special issue: Marine and coastal GIS for geomorphology, habitat mapping, and marine reserves. Marine Geodesy, 31, 223-230.

Yang HS, Zhang SY, Zhang XM, Chen PM, Tian T, Zhang T (2019) Strategic thinking on the construction of modern marine ranching in China. Journal of Fisheries of China, 43, 1255-1262. (in Chinese with English abstract) [杨红生, 章 守宇, 张秀梅, 陈丕茂, 田涛, 张涛 (2019) 中国现代化 海洋牧场建设的战略思考. 水产学报, 43, 1255-1262.]

Zhao QS, Basher Z, Costello MJ (2020) Mapping near surface global marine ecosystems through cluster analysis of environmental data. Ecological Research, 35, 327-342.

Zhao QS, Costello MJ (2019) Marine Ecosystems of the World. University of Auckland, New Zealand.

(责任编委: 郭庆华 责任编辑: 间文杰) 